\title{
Avaliando a usabilidade de websites com ênfase em usuários idosos: um estudo de caso
}

\author{
Sidney Calebe Ribeiro, UNIFEI, calebetmr@gmail.com \\ Adriana Prest Mattedi, UNIFEI, amattedi@gmail.com \\ Rodrigo Duarte Seabra, UNIFEI, rodrigo@unifei.edu.br
}

\begin{abstract}
Resumo: O estudo analisa o impacto que a idade exerce na usabilidade de websites, comparando usuários jovens e idosos, com base em critérios abordados na literatura científica. $\mathrm{O}$ envelhecimento da população e o crescente uso da internet acentua a relevância deste estudo, tendo como foco características inerentes aos idosos. O método utilizado nesta pesquisa propôs três tarefas em dois websites e a usabilidade foi investigada a partir da análise do desempenho baseado no tempo e nas percepções dos voluntários por meio de questionários. As principais conclusões alcançadas são: os idosos obtiveram desempenhos, em geral, inferiores aos jovens na realização das tarefas; a facilidade de aprendizado foi verificada e mensurada nos dois grupos de voluntários; fatores como a experiência no uso da tecnologia tornaram o desempenho dos idosos semelhante ao dos jovens.
\end{abstract}

Palavras-chave: Avaliação da Usabilidade, Usuários Idosos, Acessibilidade.

\section{Evaluating website usability with focusing on elderly users: a case study}

Abstract: The study analyzes the impact that age has on website usability, comparing young and elderly users, based on criteria discussed in the scientific literature. The aging population and the increasing use of the internet accentuate the relevance of this study, focusing on characteristics of the elderly. The method used in this research proposed three tasks on two websites and usability was investigated from the performance analysis based on time and the perceptions of the volunteers through questionnaires. The main conclusions reached are: the elderly have obtained performances, in general, lower than young people in carrying out the tasks; the ease of learning was verified and measured in both groups of volunteers; factors such as experience in the use of technology have made the elderly performance similar to the young.

Keywords: Usability Evaluation, Elderly Users, Accessibility.

\section{INTRODUÇÃO}

A internet se encontra em processo de constante evolução, trazendo novidades no que se refere ao entretenimento e na infinidade de serviços. Paralelo a este fato, também é crescente a quantidade de pessoas que acessam a internet com objetivos variados, a saber: acesso a bancos, realização de compras, interação em redes sociais, aquisição de informações em geral etc. Segundo o relatório da International Telecommunications Union (ITU, 2014), publicado anualmente, mais de $40 \%$ da população mundial, em 2014, fazia uso da internet.

Por outro lado, a população mundial está envelhecendo. Segundo dados disponibilizados pela Organização das Nações Unidas (ONU), o número de pessoas com mais de 60 anos deve dobrar até 2025. Em 2050, os idosos devem corresponder a $20 \%$ da população mundial (ONU, 2014). Além disso, parte desse grupo está 
permanecendo mais tempo no mercado de trabalho. Segundo a OECD (Organization for Economic Cooperation and Development), a oferta de jovens que ingressam no mercado de trabalho é cada vez menor, fazendo com que a força de trabalho mais velha tenha que permanecer mais tempo no mercado profissional (OECD, 2000). As organizações, por sua vez, têm grande interesse na usabilidade na web, pois esta exerce influência no desempenho do trabalho de seus colaboradores, resultando em maior (ou menor) produtividade e redução (ou aumento) de custos (DELONE e McLEAN, 2003).

No campo digital, percebe-se uma crescente inserção por parte de idosos que, até pouco tempo, se mostravam resistentes a utilizar os recursos tecnológicos. No Brasil, dados do IBOPE apontaram crescimento dos usuários idosos de internet nos últimos anos. Em 2013, por exemplo, foi constatada uma alta de 8,3\% com relação ao ano de 2012 (IBOPE, 2013).

Entretanto, existem alguns fatores fisiológicos que influenciam a utilização da internet (ou a limitação deste uso) pelos idosos, tais como: visão, cognição e deficiências físicas (BECKER, 2004). Essas dificuldades têm consequências e podem comprometer a experiência pessoal e profissional desses indivíduos.

Dessa forma, o estudo da usabilidade de websites para idosos tem ganhado importância, visando avaliar e atender melhor as necessidades deste grupo de indivíduos, tanto na vida pessoal quanto profissional. Neste contexto, esse trabalho se propõe a analisar o impacto da idade no desempenho de usuários ao utilizar websites para a realização de tarefas, dados os critérios de usabilidade definidos pela engenharia de software. Serão realizadas comparações de percepções entre pessoas de distintas idades, com base em critérios utilizados na literatura científica na área de usabilidade de websites.

\subsection{Modelos teóricos na usabilidade de websites}

O termo usabilidade é definido de maneiras distintas na literatura científica. Uma definição de simples entendimento foi oferecida por Nielsen e Loranger (2007: 16), que definiram a usabilidade como "um atributo de qualidade relacionado à facilidade de uso de algo. Mais especificamente, refere-se à rapidez com que os usuários podem aprender a usar alguma coisa, a eficiência deles ao usá-la, o quanto lembram daquilo, seu grau de propensão a erros e o quanto gostam de utilizá-la".

Muitos são os fatores que podem influenciar a usabilidade de um website. Lee (1999) descreve uma série deles: (i) as tarefas essenciais que um sistema se destina a apoiar, tais como a descoberta e compreensão de informações; (ii) variáveis do sistema, que, além de outras questões, incluem a velocidade de transmissão da internet e cores; (iii) características dos usuários, tais como fatores relativos à habilidade, conhecimento, idade, capacidades físicas e mentais; (iv) leitura, que diz respeito ao tamanho de caracteres exibidos, contraste entre o texto e o fundo da página e, inclusive, o conteúdo do website.

Flavián et al (2006) consideram a usabilidade de um website segundo alguns aspectos, sendo eles: a facilidade de compreensão da estrutura e suas funções, isto é, a interface e os conteúdos que podem ser observados pelo usuário; a simplicidade do uso nos seus estágios iniciais, ou seja, nos primeiros momentos de uso de um website; a velocidade com que os usuários podem encontrar o que estão procurando; a percepção de facilidade de navegação em termos do tempo necessário e as medidas utilizadas para obter os resultados desejados.

As pesquisas de usabilidade na web muitas vezes se dedicam a observar grupos de usuários com características específicas e em contextos bem definidos. Um exemplo 
destes estudos foi realizado por Becker (2004), que focou o uso da internet por adultos mais velhos na busca de informações relacionadas à saúde, tais como sintomas de doenças, diagnósticos, prescrição, cirurgia e recuperação. Na pesquisa, é promovida uma análise da usabilidade de vários websites que abordam esse tema de acordo com uma série de critérios, tais como: design, desempenho, tradução e complexidade de leitura. $\mathrm{O}$ trabalho concluiu que nenhum dos websites estudados alcançou índices altos em termos de usabilidade. Foi verificado, por exemplo, que cerca de $93 \%$ da amostra fazia uso de fontes pequenas, afetando negativamente a capacidade de leitura para pessoas idosas. Pouco mais da metade dos websites contava com recursos para redimensionar o conteúdo por meio do browser. Verificou-se ainda que os menus suspensos utilizados em alguns sites se apresentavam como uma barreira de usabilidade, pois alguns usuários possuíam mãos trêmulas. Foram identificados problemas também em relação à homepages longas, por exigirem alto esforço da memória frente aos conteúdos superior e inferior da página. Verificou-se, inclusive, que a falta de recursos como mapa do site e ajuda são barreiras principalmente para os usuários iniciais da internet.

Ainda a respeito da usabilidade de websites para adultos mais velhos, é relevante mencionar a pesquisa de Wagner et al (2014), que promove uma comparação do comportamento, as impressões e a conclusão de tarefas com adultos mais jovens. Para tal, é traçado um paralelo entre as reduções cognitivas que ocorrem nos indivíduos com o passar do tempo e a diminuição da capacidade de criação de modelos mentais. Esses fatores são ainda relacionados à habilidade de compreensão da estrutura de websites. Além disso, foram analisados aspectos hedônicos, por exemplo, a desorientação e a satisfação. Foi constatado na pesquisa que a idade exerce impacto sobre o desempenho de navegação em websites. Isso ocorre em função do declínio progressivo da capacidade espacial, que exerce influência na habilidade de criação de modelos mentais, interferindo, assim, na compreensão da estrutura de websites. Verificou-se, ainda, que a navegação no website também sofre impactos negativos de acordo com características comuns nos idosos, tais como o declínio da visão e da coordenação psicomotora, a redução da capacidade de atenção e a perda progressiva de memória.

A fim de selecionar na literatura os construtos de usabilidade mais relevantes, Lee e Kozar (2012) promoveram uma minuciosa análise bibliográfica, tendo o objetivo de identificar os aspectos comuns nos estudos, além de desenvolver e validar os instrumentos de medição neles utilizados. O trabalho promoveu a identificação de construtos de usabilidade, que foram agrupados e organizados. Um resultado significativo dessa pesquisa foi a identificação de dez construtos relevantes na usabilidade de websites, sendo eles: consistência, facilidade de navegação, suporte, facilidade de aprendizado, simplicidade, interatividade, facilidade de leitura, relevância de conteúdo, credibilidade e telepresença. Os critérios citados foram amplamente utilizados na literatura científica para estudar a usabilidade de websites, o que os torna pertinentes para a adoção total ou parcial em trabalhos que envolvam a usabilidade de websites.

\section{METODOLOGIA}

A pesquisa envolveu a participação de jovens e idosos, que atuaram como voluntários. Foram propostas três tarefas (T1, T2 e T3) aos participantes em dois sites diferentes. Cada tarefa concluída nos websites foi seguida por um questionário, de forma que três questionários (concernentes às tarefas) foram aplicados para cada voluntário. O objetivo foi investigar as percepções desses voluntários com relação às 
tarefas propostas dentro dos websites estudados. Além disso, um quarto questionário foi utilizado com a finalidade de traçar o perfil dos indivíduos.

A realização da pesquisa se restringe à observação de dois websites que atuam em setores diferentes. Um dos websites selecionados atua no ramo de e-commerce, já bem estabelecido no Brasil (www.americanas.com.br), e o outro é um site governamental cujos serviços possuem grande foco para idosos (www.previdencia.gov.br). A intenção dessa abordagem foi verificar se o website comercial teria resultados melhores que um website do governo, devido a questões como investimento para geração de receita e obtenção de lucro.

Para a realização da pesquisa, foram selecionados na literatura (LEE e KOZAR, 2012; WAGNER et al, 2014) os aspectos de usabilidade da web relevantes para pessoas idosas. Esses aspectos abrangem tanto questões hedônicas quanto utilitárias. Desse modo, faz-se necessário levar em consideração as limitações progressivas que ocorrem nos indivíduos com a idade mais avançada.

Cada tarefa realizada nos websites envolveu a resposta de um questionário respectivo a esta tarefa. $\mathrm{O}$ objetivo foi avaliar os critérios de usabilidade definidos no Quadro 1.

Quadro 1: Critérios de usabilidade e suas definições.

\begin{tabular}{|c|c|}
\hline $\begin{array}{l}\text { Facilidade de } \\
\text { navegação }\end{array}$ & $\begin{array}{l}\text { Capacidade de o website fornecer técnicas de interação e de navegação alternativas (LEE e KOZAR, } \\
\text { 2012). }\end{array}$ \\
\hline Simplicidade & Disponibilização do mínimo de conteúdo e funções dentro de um website (LEE e KOZAR, 2012). \\
\hline Suporte & $\begin{array}{l}\text { Mecanismos de informação e suporte adicionais disponíveis para aprimorar a experiência de uso do } \\
\text { website (LEE e KOZAR, 2012). }\end{array}$ \\
\hline $\begin{array}{l}\text { Facilidade de } \\
\text { Aprendizado }\end{array}$ & $\begin{array}{l}\text { Facilidade de aprender a funcionalidade principal do website e adquirir proficiência para completar } \\
\text { as tarefas (LEE e KOZAR, 2012). }\end{array}$ \\
\hline $\begin{array}{l}\text { Facilidade de } \\
\text { Leitura }\end{array}$ & $\begin{array}{l}\text { Medida em que os componentes do website são bem organizados, fáceis de ler e entender (LEE e } \\
\text { KOZAR, 2012). }\end{array}$ \\
\hline Desorientação & $\begin{array}{l}\text { Sensação de estar perdido. Ocorre quando um usuário perde o senso de localização e direção de um } \\
\text { documento não linear (AHUJA e WEBSTER, 2001; CONKLIN, 1987). }\end{array}$ \\
\hline Desempenho & Eficiência e eficácia na utilização de uma tecnologia (COURSARIS e KIM, 2011). \\
\hline Satisfação & $\begin{array}{l}\text { Relaciona-se com a satisfação subjetiva dos usuários com o uso da tecnologia (COURSARIS et al, } \\
\text { 2012). }\end{array}$ \\
\hline
\end{tabular}

O conjunto de questionários foi composto por um grupo inicial de questões, com a finalidade de traçar o perfil de cada voluntário. O conteúdo desse grupo envolveu aspectos como: idade, tempo de utilização da internet, finalidade desse uso, grau de conhecimento da tecnologia, necessidade de auxílio para sua utilização, além de escolaridade e profissão. Essa etapa inicial teve a intenção de verificar se os aspectos culturais ou habituais dos voluntários influenciariam no desempenho ao utilizar os websites, além da idade.

Após cada tarefa ser realizada, foi aplicado um questionário contendo 23 perguntas com respostas segundo a escala Likert, que englobavam os critérios de usabilidade considerados no estudo. Foi abordada também uma questão sobre a conclusão das tarefas, para servir de ferramenta de avaliação, juntamente com a análise do tempo demandado para realização das mesmas. Possíveis dificuldades com o mouse e/ou teclado também foram englobadas no questionário para avaliar impactos da redução da coordenação motora com a idade. Cada questionário foi finalizado com o convite ao voluntário para adicionar comentários sobre a experiência de utilizar cada website. As questões relativas aos critérios de usabilidade definidos encontram-se listadas no Quadro 2. 


\subsection{Pesquisa de campo}

A pesquisa fez observação de indivíduos de dois grupos de idades muito distintas, abrangendo tanto pessoas jovens, na faixa de 17 a 21 anos, quanto pessoas mais velhas, com idades variando entre 62 e 77 anos. Houve, então, a composição de dois grupos: um composto pelos jovens e outro pelos idosos (acima de 60 anos). Ambos os grupos foram compostos por 13 voluntários.

Quadro 2: Questões relativas aos critérios de usabilidade.

\begin{tabular}{|c|c|}
\hline Critério & Questões \\
\hline $\begin{array}{l}\text { Facilidade de } \\
\text { aprendizado }\end{array}$ & $\begin{array}{l}\text { 1) As informações disponíveis no site são facilmente compreendidas? } \\
\text { 2) Eu posso recordar facilmente os passos para realizar a mesma tarefa novamente? }\end{array}$ \\
\hline Simplicidade & $\begin{array}{l}\text { 3) Como você avalia o caminho percorrido na tarefa proposta? } \\
\text { 4) As informações apresentadas no site estão em excesso? } \\
\text { 5) Eu posso compreender rapidamente a maioria das informações/instruções dentro do site? } \\
\text { 6) Como você avalia a simplicidade para realizar uma compra no site? }\end{array}$ \\
\hline Suporte & $\begin{array}{l}\text { 7) Você teve alguma dúvida ou dificuldade para a realização da tarefa dentro do site? } \\
\text { 8) Você procurou informações adicionais no site para auxiliar a realização da tarefa? } \\
\text { 9) O site forneceu informações adicionais para esclarecer suas dúvidas/dificuldades ou algum } \\
\text { mecanismo de ajuda? } \\
\text { 10) Como você avalia o suporte oferecido pelo site para a realização da tarefa? }\end{array}$ \\
\hline Desorientação & $\begin{array}{l}\text { 11) Você considera que o caminho percorrido na tarefa foi intuitivo? } \\
\text { 12) Enquanto estava navegando no site, você se sentiu: }\end{array}$ \\
\hline Satisfação & $\begin{array}{l}\text { 13) Você se sentiu satisfeito ao realizar a tarefa no site? } \\
\text { 14) Caso você tenha se sentido insatisfeito ou muito insatisfeito, informe o motivo. }\end{array}$ \\
\hline $\begin{array}{l}\text { Facilidade de } \\
\text { leitura }\end{array}$ & $\begin{array}{l}\text { 15) A linguagem utilizada no site foi de fácil entendimento? } \\
\text { 16) Os espaçamentos entre as palavras e imagens tornou a leitura fácil? } \\
\text { 17) As cores utilizadas no site facilitaram a visualização? } \\
\text { 18) O tamanho das letras do site facilitou a visualização? } \\
\text { 19) A quantidade de informações exibidas coube de modo adequado em cada página? } \\
\text { 20) Como você avalia o site em relação à facilidade de leitura? }\end{array}$ \\
\hline Desempenho & 21) Você conseguiu concluir a tarefa? \\
\hline $\begin{array}{l}\text { Facilidade de } \\
\text { navegaçãa }\end{array}$ & $\begin{array}{l}\text { 22) Você teve alguma dificuldade em utilizar o mouse e/ou o teclado para realizar a tarefa proposta } \\
\text { dentro do site? } \\
\text { 23) Você deseja acrescentar algum comentário sobre sua experiência ao navegar no site? }\end{array}$ \\
\hline
\end{tabular}

O perfil dos jovens era semelhante com relação à formação, sendo todos estudantes universitários. A média de tempo de uso da internet desse grupo é de 8,8 anos. Os interesses na internet apresentados por eles variaram entre estudo, entretenimento e acesso à informação. $\mathrm{O}$ perfil dos adultos mais velhos com relação ao tempo de uso da internet foi de 8,9 anos, em média. Quanto à formação, a maioria possui ensino superior ou pós-graduação. Os interesses na internet, além dos apresentados pelos jovens, englobou também o uso para o trabalho.

Todos os participantes foram convidados à realização de três tarefas bem definidas, envolvendo atividades simples como o download de um documento no website e a realização de uma tarefa mais complexa, como efetuar uma compra online. Posteriormente, eles foram convidados a responder um questionário, no qual relataram as dificuldades/facilidades encontradas nas tarefas realizadas sob o ponto de vista dos critérios de usabilidade definidos.

A disposição das tarefas realizadas nos websites ocorreu da seguinte maneira: a primeira tarefa (T1) solicitou a compra de um telefone celular no website de $e$ commerce. A segunda tarefa (T2) envolveu a realização de um download de um documento específico no website governamental. Finalmente, a terceira tarefa (T3) foi muito semelhante à $\mathrm{T} 1$, abordando a compra de um dicionário no mesmo website da primeira tarefa executada. A tarefa T3 foi propositalmente semelhante à tarefa $\mathrm{T} 1$, pois 
teve a intenção de avaliar se, ao repetir uma tarefa, o usuário seria capaz de demonstrar possíveis questões de aprendizado, apresentando, assim, melhor desempenho. As tarefas T1 e T3 se diferenciaram apenas com relação ao produto a ser comprado no website.

Os voluntários receberam as orientações contendo os passos necessários para a execução das tarefas. Antes de iniciar cada tarefa, primeiramente, cada voluntário leu todas as orientações e, posteriormente, partiu para a execução, computando o tempo de início e de fim. Em seguida, o participante respondeu um questionário respectivo àquela tarefa. $\mathrm{O}$ pesquisador não exerceu qualquer influência nos participantes com relação à execução das tarefas, bem como qualquer auxílio sobre dúvidas de como utilizar os websites, encontrar informações ou executar passos. As dúvidas enfrentadas sobre a utilização do website eram de responsabilidade do voluntário.

\section{DISCUSSÃO DOS RESULTADOS}

Com relação à $\mathrm{T} 1$ e a respeito dos voluntários idosos, é relevante mencionar alguns resultados. Aqueles que avaliaram negativamente as questões concernentes à facilidade de leitura obtiveram médias de tempo maiores, se comparados aos demais do mesmo grupo. Para os indivíduos que consideraram a linguagem do website de difícil entendimento, foram necessários, em média, 6,2 minutos para a conclusão da tarefa. Já os que tiveram uma visão positiva nessa questão, levaram, em média, 5,5 minutos. De modo semelhante, houve desempenho inferior para os voluntários que consideraram que as cores utilizadas no website e os espaçamentos entre palavras e imagens não facilitaram a visualização. Os voluntários que informaram que os tamanhos das fontes não facilitaram a visualização necessitaram de 5,9 minutos, em média, para realizar a compra. Porém, os que manifestaram que as fontes facilitaram a visualização concluíram a tarefa com 5,1 minutos, em média. Foi verificado um desempenho de tempo em torno de três vezes maior para os indivíduos que avaliaram o website muito fácil de ler frente aos que consideraram difícil de ler. Os primeiros gastaram 2 minutos, em média, para concluir a tarefa, ao passo que os demais, 6 minutos. Esses resultados revelam que o critério exerce influência no desempenho na conclusão de tarefas. Para melhor atender os adultos mais velhos que possuem limitações inerentes à idade, é clara a necessidade de se atentar para algumas de suas características particulares: é importante que os websites utilizem tamanhos de fontes, cores e espaçamentos adequados, sobretudo uma linguagem de mais fácil assimilação.

Observando o tempo reduzido apresentado ao repetir a mesma tarefa (T3), foi verificado que o aprendizado reflete no desempenho e, por consequência, na melhora da usabilidade. É importante verificar que a facilidade de aprendizado relaciona-se ao contato que o usuário tem com o website. Quanto mais este usuário acessar o mesmo website, maior será seu aprendizado e, por consequência, maior a usabilidade percebida.

O tempo alto investido na interação do usuário com o website influencia também na sua satisfação. Foi verificado que os indivíduos idosos que se manifestaram insatisfeitos na realização da tarefa levaram, em média, 10 minutos para concluí-la, diferentemente dos indivíduos que se manifestaram como satisfeitos, que levaram, em média, 5,5 minutos.

Com relação à desorientação dentro do website, foi verificado que aqueles que manifestaram opinião como totalmente perdido durante a navegação levaram, em média, 8 minutos para realizar a compra. Já aqueles que permaneceram totalmente orientados, levaram 5,5 minutos para concluí-la.

A simplicidade para a realização da tarefa também exerceu influência nos tempos gastos em sua conclusão. Verificou-se que os indivíduos que avaliaram o 
caminho percorrido na realização da compra como fácil necessitaram de 4 minutos, em média, para concluí-la. Já os que avaliaram como difícil, levaram o dobro do tempo. Esta diferença de tempo também foi verificada nos usuários que informaram que não podiam compreender rapidamente as informações/instruções dentro do website. Foi verificado igualmente um desempenho alto nos voluntários idosos que informaram que o website era muito simples para realizar a compra, levando, em média, 2 minutos; já aqueles que informaram que a simplicidade era média levaram 5,9 minutos, em média.

Analisando a Tarefa 2 realizada pelo grupo dos adultos mais velhos, novamente foi possível verificar uma relação entre desempenho e os critérios de usabilidade. Primeiramente, foi notado que os indivíduos que avaliaram de modo negativo as questões relativas à simplicidade do website levaram tempos maiores para a execução do download. Aqueles que consideraram que o caminho percorrido na execução da Tarefa 2 era fácil realizaram o download em 6,1 minutos, em média. Já os voluntários que responderam a questão como difícil, concluíram a tarefa em 7,2 minutos. Para os indivíduos que avaliaram a simplicidade de se executar o download como fácil, o tempo na conclusão foi de 6,3 minutos, em média. Para aqueles que avaliaram o download como muito difícil, foi necessária uma média de 8 minutos para a conclusão da tarefa.

Nas questões que visavam avaliar a facilidade de leitura, foi verificada também, no website governamental, a influência no desempenho dos participantes. Dentro do grupo de idosos, aqueles que informaram que o website era muito fácil de ler gastaram, em média, 1 minuto para concluir a tarefa. No outro extremo, aqueles que informaram que o website era difícil de ler necessitaram de 8 minutos, em média, para concluir a tarefa. Foi também alta a diferença dentre aqueles que informaram que as informações não couberam de modo adequado em cada página. Estes gastaram 6,6 minutos para concluir a tarefa, em média, frente aos 12 minutos daqueles que avaliaram de forma negativa esse critério. O tamanho das fontes também influenciou o desempenho dos voluntários na conclusão do download. Aqueles que opinaram que o tamanho das fontes facilitou a visualização concluíram a tarefa em 6,1 minutos, em média, ao passo que para aqueles que não tiveram essa mesma opinião, foram necessários, em média, 7,1 minutos. Semelhante ao que foi notado na Tarefa 1, os fatores que compõem a facilidade de leitura tem intensa ligação com o desempenho do usuário idoso no website.

Os adultos mais velhos que permaneceram orientados durante todo o tempo da navegação levaram, em média, 6,6 minutos para realizar o download. Já os que reportaram algum problema de orientação levaram 7,1 minutos para a conclusão da tarefa.

Os tamanhos das fontes utilizadas aparentam ter contribuído para causar dúvidas ou dificuldades nos usuários idosos. Cerca de $83 \%$ dos idosos que reportaram dúvida/dificuldade no website comercial também informaram que os tamanhos das fontes não facilitaram a visualização. No website governamental, o número foi ainda maior, correspondendo a aproximadamente $88 \%$.

Relacionando as variáveis facilidade de leitura (Q16) com simplicidade de compra (Q6), pode-se perceber, pelo gráfico de dispersão mostrado na Figura 1, um alto grau de associação entre estas duas variáveis, evidenciando que a facilidade de leitura de um website está ligada à simplicidade que o usuário encontra ao realizar as tarefas nele dispostas. $O$ coeficiente de correlação encontrado entre essas variáveis foi de 0,71 .

Em torno de $75 \%$ dos adultos mais velhos com experiência na internet igual ou superior a 10 anos obtiveram um desempenho de até 1,9 minutos, em média, nas três tarefas. Verifica-se semelhança ao tempo dos voluntários jovens, levando em conta que a média de tempo para este grupo foi de aproximadamente 2,3 minutos. Os idosos em 
questão também estabeleceram avaliações melhores sobre a usabilidade dos websites se comparadas aos demais com menos anos de experiência com a internet. De modo semelhante, aqueles que declararam possuir conhecimento alto de internet obtiveram médias de tempo menores que a média total. Com tempos médios em torno de 3,8 minutos, verifica-se que, além do desempenho, as percepções em geral de usabilidade estão ligadas aos conhecimentos e a experiência com a internet.

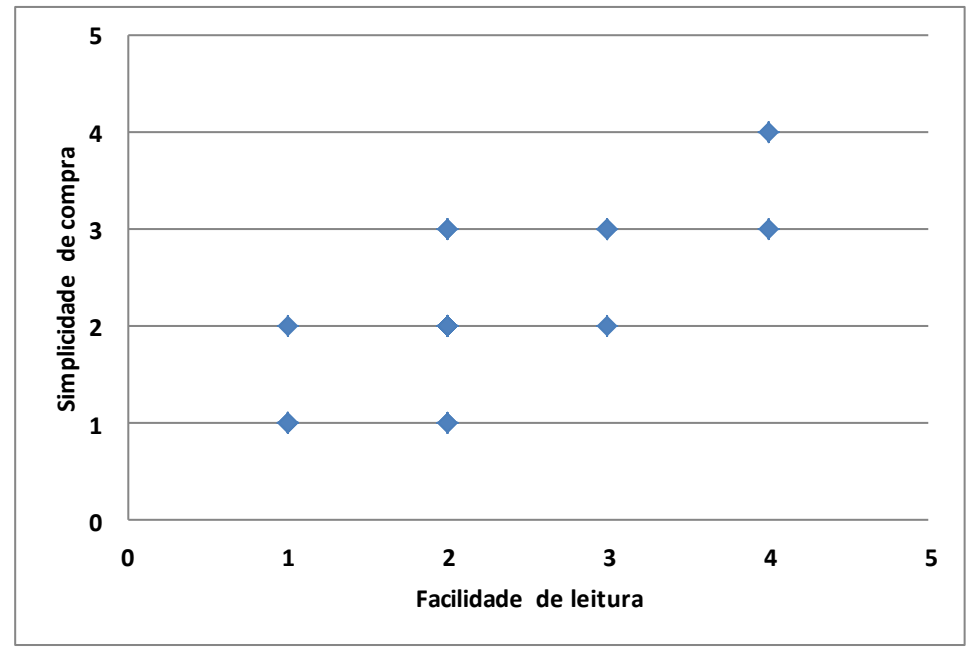

Figura 1: Relação entre facilidade de leitura (Q18) e simplicidade de compra (Q6) obtida nos questionários referentes às tarefas T1 e T2.

Os indivíduos idosos que permaneceram orientados durante todo o tempo de navegação no website comercial, em geral, estabeleceram avaliações positivas com relação a vários critérios de usabilidade. Estes voluntários reportaram que: (i) as informações disponíveis dentro do website eram facilmente compreendidas; (ii) podiam recordar facilmente os passos para realizar a tarefa novamente; (iii) não enfrentaram nenhuma dúvida ou dificuldade para realizar a tarefa; (iv) consideravam o caminho para a conclusão da tarefa intuitivo; (v) avaliaram a linguagem do website de fácil entendimento. Desses voluntários, $67 \%$ informaram que já haviam feito compras em websites de e-commerce anteriormente. É possível concluir que a orientação do usuário no website depende de uma série de fatores de usabilidade, tais como: facilidade de aprendizado, simplicidade, facilidade de navegação e facilidade de leitura. Verifica-se ainda que a experiência do usuário também exerce influência nas suas percepções de usabilidade.

\section{CONSIDERAÇÕES FINAIS}

Este trabalho buscou verificar o impacto da idade no desempenho de idosos no uso da internet, sob o ponto de vista dos requisitos de usabilidade, em comparação com pessoas jovens. Percebeu-se que, de fato, o desempenho das pessoas com idades acima de 60 anos é inferior. Os adultos mais velhos gastaram mais que o dobro do tempo para concluírem as mesmas tarefas realizadas pelos jovens. Fica evidente, portanto, que a idade exerce influência na usabilidade de websites, devido às reduções progressivas das capacidades cognitivas e motoras.

Existem, porém, outros fatores que atuam em conjunto na redução (ou aumento) da usabilidade de websites. A menor usabilidade relaciona-se também a questões como a experiência e o conhecimento de internet dos idosos, refletindo no seu desempenho ao 
utilizar o website. A satisfação é influenciada pelo conteúdo do website, além do tempo empregado para conseguir concluir as tarefas nele contidas, de modo que quanto maior o desempenho, maior a satisfação.

A desorientação dos idosos foi alta, exercendo influência também nos desempenhos inferiores e na dificuldade de navegação. Porém, estas questões não se relacionam apenas com reduções de capacidades cognitivas, pois dependem de outros critérios que atuam de modo conjunto, incluindo a facilidade de leitura. Os websites estudados estão despreparados para atender os adultos mais velhos adequadamente. Os tamanhos das fontes e as cores impactaram no desempenho, causaram dúvidas e dificuldades nesses indivíduos.

Diferentemente do que é verificado em alguns trabalhos científicos, notou-se a ausência de interesse dos idosos por suporte. A desconfiança da eficácia dos serviços do gênero, oferecidos pelos websites no Brasil, em geral, pode ter ocasionado o desinteresse. A pesquisa ainda foi capaz de contabilizar a facilidade de aprendizado de um website verificando que a repetição de tarefas gera aumento de desempenho com base no tempo. Neste âmbito, quanto maior o acesso a um mesmo website, maior o aprendizado e, por consequência, a usabilidade.

Conclui-se que a idade não é o único fator que influencia a usabilidade de websites. Outras questões estão relacionadas à usabilidade na web e estas também precisam ser levadas em consideração ao planejar e implementar websites que procurem melhor atender esse público.

Percebeu-se que a ausência de motivação pessoal para executar certas atividades é capaz de influenciar os resultados da investigação de critérios de usabilidade, tais como a satisfação. O critério em questão pode, todavia, ser investigado através de questões direcionadas à irritação com que o usuário apresenta ao efetuar uma tarefa em um dado website. Desta maneira, reduz-se a influência do interesse pessoal do voluntário em suas avaliações a respeito da satisfação.

Em pesquisas futuras, é relevante estabelecer um número reduzido de critérios de usabilidade para efetuar uma investigação mais aprofundada. O uso de questionários longos configura uma limitação deste estudo, pois essa característica pode desmotivar os voluntários e influenciar suas respostas.

Esta pesquisa foi capaz de analisar a facilidade de aprendizado por meio da repetição de uma mesma tarefa em pequenos intervalos. Estudos futuros podem estudar esse critério com base em intervalos maiores, dias ou semanas. Deste modo, é possível avaliar como esses fatores, incluindo as reduções de memória e cognição, influenciam na vida dos idosos.

Há ainda demanda pela pesquisa da usabilidade com foco em outras características presentes no cotidiano dos indivíduos mais velhos. É relevante analisar a usabilidade que os adultos mais velhos possuem ao utilizar outros equipamentos, como smartphones e tablets. Estes dispositivos possuem particularidades envolvendo tamanhos de tela reduzidos, distintos sistemas operacionais embarcados, telas sensíveis ao toque etc., que podem se apresentar como barreiras para o público mais idoso.

\section{REFERÊNCIAS}

AHUJA, J. S., WEBSTER, J. Perceived disorientation: an examination of a new measure to assess web design effectiveness. Interacting with computers, v. 14, n. 1, p. 15-29, 2001. 
BECKER, S. A. A study of web usability for older adults seeking online health resources. ACM Transactions on Computer-Human Interaction (TOCHI), v. 11, n. 4, p. 387-406, 2004.

CONKLIN, J. Hypertext: A survey and introduction. IEEE Computer, v. 20, n. 9, p. 17-41, 1987.

COURSARIS, C. K., HASSANEIN, K., HEAD, M. M., BONTIS, N. The impact of distractions on the usability and intention to use mobile devices for wireless data services. Computers in Human Behavior, v. 28, n. 4, p. 1439-1449, 2012.

COURSARIS, C. K., KIM, D. J. A meta-analytical review of empirical mobile usability studies. Journal of usability studies, v. 6, n. 3, p.117-171, 2011.

DELONE, W. H., McLEAN, E. R. The DeLone and McLean model of information systems success: a ten-year update. Journal of Management Information Systems, v. 19, n. 4, p. 9-30, 2003.

FLAVIÁN, C., GUINALÍU, M., GURREA, R. The role played by perceived usability, satisfaction and consumer trust on website loyalty. Information \& Management, v. 43, n. 1, p. 1-14, 2006.

IBOPE. Instituto Brasileiro de Opinião e Estatística. 28\% dos idosos se mantêm atualizados com as novas tecnologias, 2013. Disponível em: http://www.ibope.com.br/pt-br/noticias/Paginas/28-dos-idosos-se-mantem-atualizadoscom-as-novas-tecnologias.aspx". Acessado em: outubro, 2015.

ITU. International Telecommunication Union. Measuring the Information Society Report. p. 252, 2014.

LEE, A. T. Web usability. ACM SIGCHI Bulletin, v. 31, n. 1, p.38-40, 1999.

LEE, Y., KOZAR, K. A. Understanding of website usability: Specifying and measuring constructs and their relationships. Decision Support Systems, v. 52, n. 2, p. 450-463, 2012.

NIELSEN, J., LORANGER, H. Usabilidade na Web. Rio de Janeiro: Elsiever, 2007.

OECD. Ageing of the Labour Force in OECD Countries: Economic and Social Consequences, 2000.

ONU. Organização das Nações Unidas. Em 2050, idosos serão dois bilhões de pessoas ou $20 \%$ de toda a população mundial, 2014. Disponível em: http://nacoesunidas.org/em-2050-idosos-serao-dois-bilhoes-de-pessoas-ou-20-de-todaa-populacao-mundial-diz-onu/. Acessado em: outubro, 2015.

WAGNER, N., HASSANEIN, K., HEAD, M. The impact of age on website usability. Computers in Human Behavior, v. 37, p. 270-282, 2014. 